\title{
Erratum
}

\section{Erratum to "Short Implants in Partially Edentulous Maxillae and Mandibles: A 10 to 20 Years Retrospective Evaluation"}

\author{
Diego Lops, ${ }^{1}$ Eriberto Bressan, ${ }^{2}$ Gianluca Pisoni, ${ }^{1}$ Niccolò Cea, ${ }^{1}$ \\ Boris Corazza, ${ }^{2}$ and Eugenio Romeo ${ }^{3}$ \\ ${ }^{1}$ Department of Prosthodontics, S. Paul Hospital Dental Clinic, School of Dentistry, University of Milan, 20142 Milan, Italy \\ ${ }^{2}$ Department of Implant Dentistry, Padova University Institute of Clinical Dentistry, 35020 Padova, Italy \\ ${ }^{3}$ Department of Implant Dentistry, S. Paul Hospital Dental Clinic, School of Dentistry, University of Milan, 20142 Milan, Italy
}

Correspondence should be addressed to Diego Lops; diego.lops@guest.unimi.it

Received 31 March 2013; Accepted 29 May 2013

Copyright (c) 2013 Diego Lops et al. This is an open access article distributed under the Creative Commons Attribution License, which permits unrestricted use, distribution, and reproduction in any medium, provided the original work is properly cited.

TABLE 1: Implant distribution by diameter, length, and type.

\begin{tabular}{lcc}
\hline Length & Diameter & Number \\
\hline \multirow{3}{*}{$8 \mathrm{~mm}$} & $3.3 \mathrm{~mm}$ & 21 \\
& $4.1 \mathrm{~mm}$ & 66 \\
& $4.8 \mathrm{~mm}$ & 21 \\
\hline \multirow{3}{*}{$10 \mathrm{~mm}$} & & 108 \\
\hline \multirow{3}{*}{} & $3.3 \mathrm{~mm}$ & 33 \\
& $4.1 \mathrm{~mm}$ & 89 \\
& $4.8 \mathrm{~mm}$ & 37 \\
\hline
\end{tabular}


TABLE 4: Short and standard implants distribution: compliances and failures.

\begin{tabular}{|c|c|c|c|c|}
\hline Site & Implant size $(\mathrm{mm})$ & Type of prosthesis & Cause of compliance & Cause of failure \\
\hline 24 & $3.3 \times 8$ & FCD & - & Mobility due to severe peri-implantitis \\
\hline 36 & $4.1 \times 8$ & FPD & - & Mobility due to severe peri-implantitis \\
\hline 16 & $4.1 \times 8$ & FPD & - & Mobility due to severe peri-implantitis \\
\hline 15 & $4.1 \times 8$ & FPD & - & Mobility due to severe peri-implantitis \\
\hline 46 & $4.8 \times 8$ & ST & Pathologic peri-implant bone resorption & - \\
\hline 35 & $4.1 \times 8$ & FPD & Successfully treated peri-implantitis & - \\
\hline 24 & $4.1 \times 8$ & ST & Successfully treated peri-implantitis & - \\
\hline 15 & $4.1 \times 8$ & FPD & Successfully treated peri-implantitis & - \\
\hline 25 & $4.1 \times 8$ & ST & Successfully treated peri-implantitis & - \\
\hline 24 & $4.1 \times 8$ & FCD & Pathologic peri-implant bone resorption & - \\
\hline 16 & $4.1 \times 8$ & ST & Pathologic peri-implant bone resorption & - \\
\hline 24 & $4.1 \times 8$ & FPD & Successfully treated peri-implantitis & - \\
\hline 45 & $4.1 \times 8$ & FPD & Pathologic peri-implant bone resorption & - \\
\hline 16 & $4.8 \times 10$ & ST & - & Mobility due to severe peri-implantitis \\
\hline 24 & $4.1 \times 10$ & FCD & - & Mobility due to severe peri-implantitis \\
\hline 25 & $3.3 \times 10$ & FPD & - & Mobility due to severe peri-implantitis \\
\hline 36 & $4.1 \times 10$ & FPD & - & Mobility due to severe bone resorption \\
\hline 37 & $4.1 \times 10$ & FPD & Successfully treated peri-implantitis & - \\
\hline 14 & $4.1 \times 10$ & FPD & Pathologic peri-implant bone resorption & - \\
\hline 25 & $4.8 \times 10$ & FPD & Successfully treated peri-implantitis & - \\
\hline 16 & $4.1 \times 10$ & ST & Successfully treated peri-implantitis & - \\
\hline 46 & $4.1 \times 10$ & FPD & Successfully treated peri-implantitis & - \\
\hline 25 & $4.1 \times 10$ & FPD & Successfully treated peri-implantitis & - \\
\hline 35 & $4.1 \times 10$ & ST & Pathologic peri-implant bone resorption & - \\
\hline 25 & $4.1 \times 10$ & ST & Pathologic peri-implant bone resorption & - \\
\hline 34 & $4.1 \times 10$ & FPD & Pathologic peri-implant bone resorption & - \\
\hline 16 & $4.8 \times 10$ & FPD & Pathologic peri-implant bone resorption & - \\
\hline 44 & $4.1 \times 10$ & FPD & Successfully treated peri-implantitis & - \\
\hline
\end{tabular}

Tooth numbers: 14 = maxillary right first premolar, $15=$ maxillary right second premolar, 16 = maxillary right first molar, $24=$ maxillary left first premolar, 25 = maxillary left second premolar, $35=$ mandibular right second premolar, $36=$ mandibular left first molar, $37=$ mandibular left second molar $44=$ mandibular right first premolar, and $46=$ mandibular right first molar.

$\mathrm{ST}=$ single tooth prosthesis, $\mathrm{FCD}=$ fixed complete dentures, $\mathrm{PFD}=$ partial fixed dentures. 


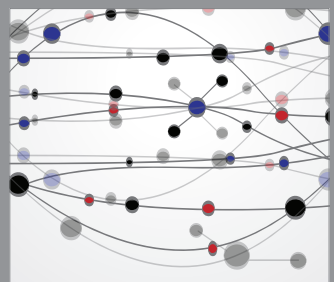

The Scientific World Journal
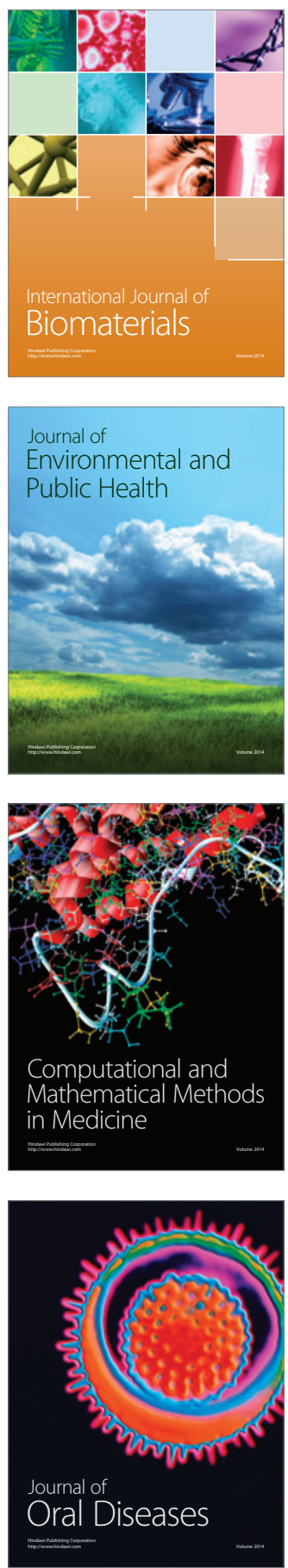
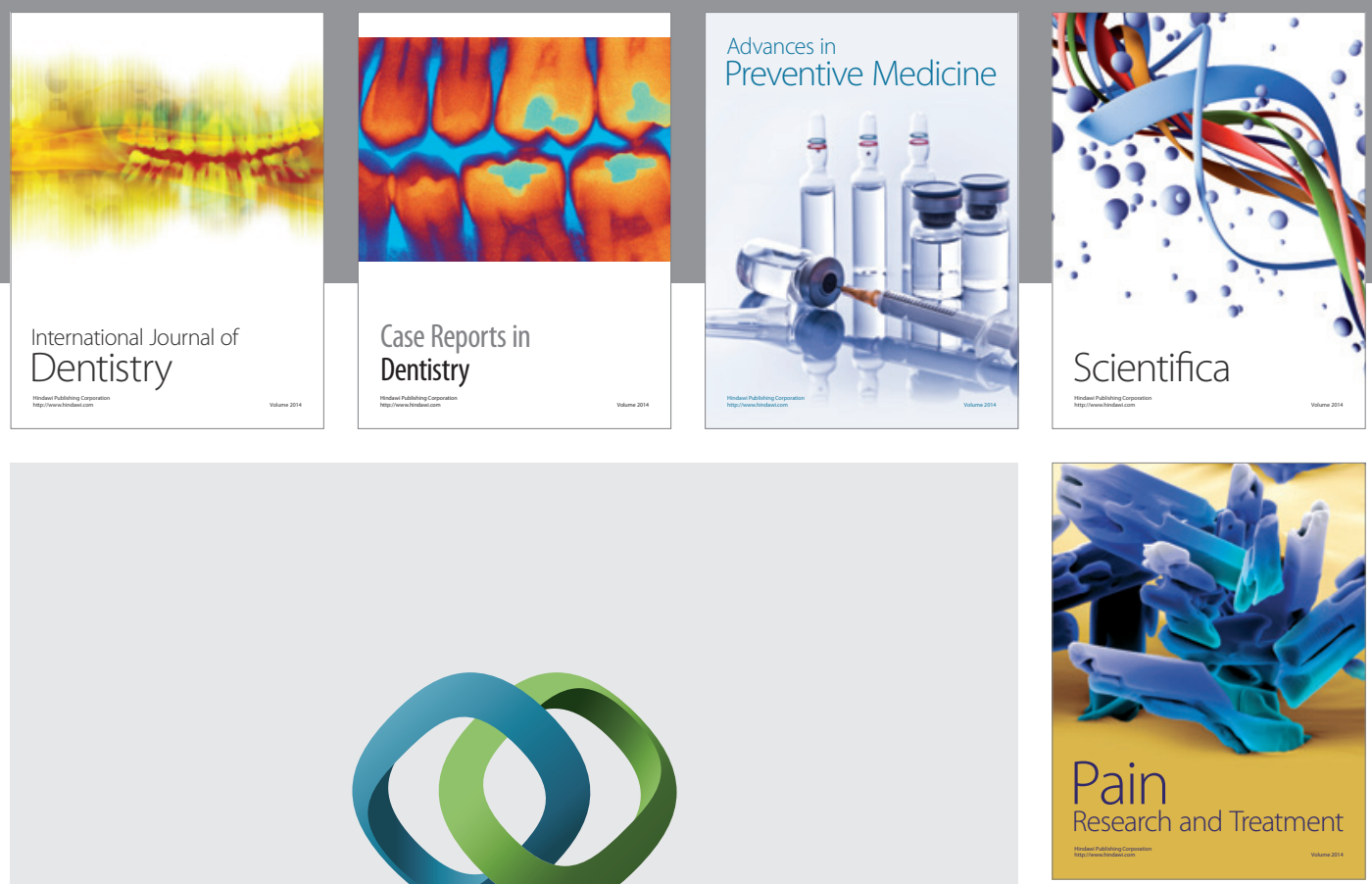

\section{Hindawi}

Submit your manuscripts at

http://www.hindawi.com
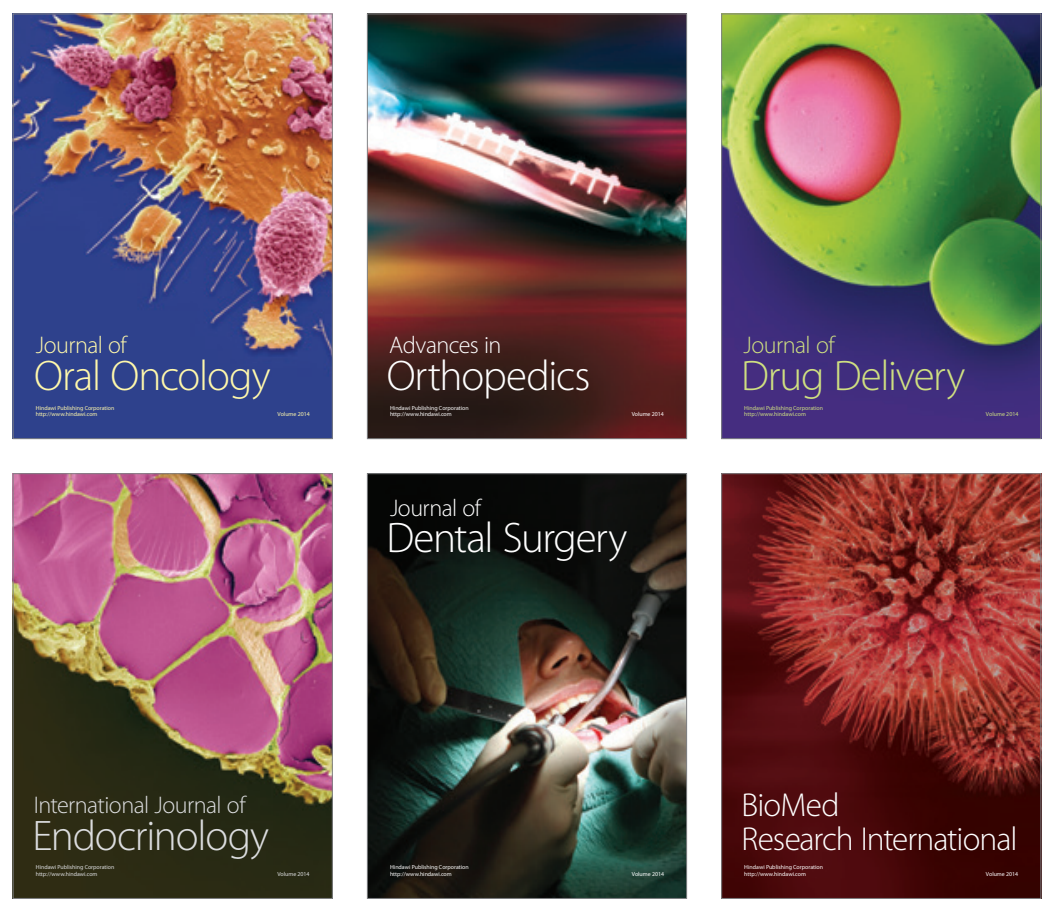

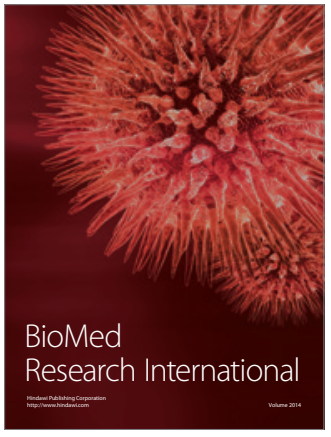

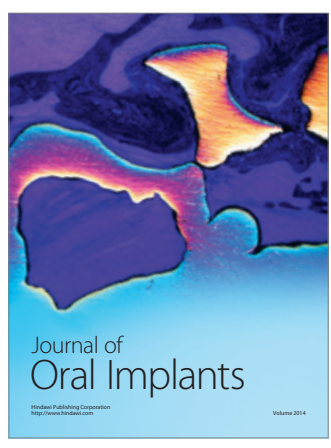
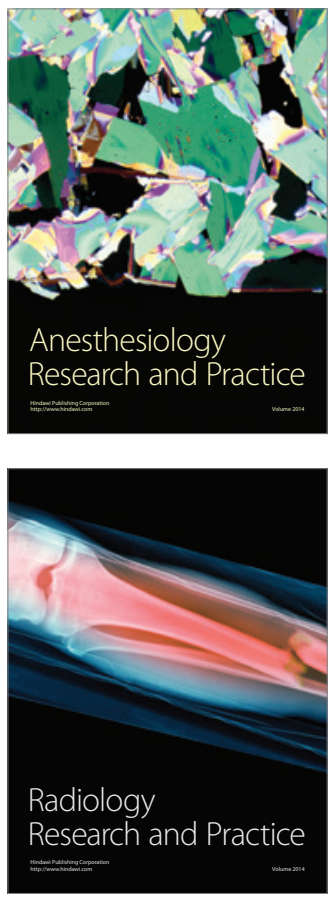